were incontinent had a 1.3-fold increased risk of becoming incontinent themselves. The risk was increased to 1.9-fold in the case of severe incontinence. In some cases, data were available for three generations: women whose mothers and grandmothers were incontinent had a 2.4-fold higher risk of developing the condition. In addition, there was a relative risk of 1.6 in women whose older sisters were sufferers. Although these increased risks applied to stress incontinence or mixed incontinence, there were too few participants to allow conclusions to be drawn about the risk of urge incontinence.

In conclusion, this study adds familial predisposition to the list of known risk factors for female urinary incontinence.

Original article Hannestad YS et al. (2004) Familial risk of urinary incontinence in women: population based cross sectional study. BMJ 329: 889-891

\section{Salvage surgery in prostate cancer: assessment of rectal wall invasion}

Extensive pelvic surgery, including total pelvic exenteration (TPE) or cystoprostatectomy, is used as a palliative treatment in some patients with locally advanced prostate cancer. The success of this approach relies on the accurate assessment of the extent of invasion into the bladder or rectum. It is not clear, however, which imaging modality is the most appropriate. In a recent study, Leibovici et al. have compared two common methods-transrectal ultrasound (TRUS) and magnetic resonance imaging (MRI) - in the detection of rectal wall involvement.

This retrospective study included 40 men who had undergone TPE $(n=16)$ or cystoprostatectomy $(n=24)$ as salvage therapy for locally advanced prostate cancer. The patients were assessed preoperatively using TRUS, MRI or both. The sensitivity and specificity of the two methods were calculated with reference to histologic findings, positive surgical margins or recurrence of disease in the rectal wall during a median follow-up of 18.6 months.

Although MRI had specificity of $100 \%$ for the detection of rectal wall involvement, its sensitivity was low at $55 \%$ and so this method is associated with a risk of false-negative results. In contrast, TRUS was highly sensitive $(93 \%)$ and fairly specific (87\%), with an overall accuracy of $89 \%$.

Original article Leibovici D et al. (2005) Transrectal

ultrasound versus magnetic resonance imaging for detection of rectal wall invasion by prostate cancer. Prostate 62: 101-104

\section{Towards a treatment for low sperm motility in men with spinal cord injury}

Spinal cord injury (SCI) not only causes problems with sexual function but also affects the characteristics of the patient's sperm; men with $\mathrm{SCl}$ are more likely to have high sperm concentrations and sperm motility tends to be lower. Seminal plasma from men with $\mathrm{SCl}$ has been shown to reduce sperm motility in samples from normal men, and sperm collected from the vas deferens of $\mathrm{SCl}$ patients has higher motility than sperm collected from their ejaculates. These findings indicate that factors in the seminal plasma itself are at least partly responsible for the reduced sperm motility in $\mathrm{SCl}$. Indeed, studies have shown high levels of certain inflammatory cytokines in the seminal plasma of SCI patients.

These observations prompted Cohen et al. to investigate whether inactivation of three cytokines -interleukin (IL)-6, IL-1- $\beta$ and tumor necrosis factor (TNF)- $\alpha$-might restore sperm motility. In their recent study, the authors collected semen from 17 men with $\mathrm{SCl}$ and added monoclonal antibodies to IL-6, IL-1- $\beta$ and TNF- $\alpha$. After 1.0 to 1.5 hours incubation at room temperature, mean sperm motility in the treated samples was higher than that of the untreated samples. This difference was statistically significant for samples that had been exposed to all three monoclonal antibodies at once (mean sperm motility $36.0 \% \pm 4.0 \%$ versus $21.2 \% \pm 4.0 \%, P<0.01$ ). The degree of improvement varied according to the pre-treatment sperm motility of the samples.

The authors note that this is the first example of a treatment for low sperm motility in this patient group.

Original article Cohen DR et al. (2004) Sperm motility in men with spinal cord injuries is enhanced by inactivating cytokines in the seminal plasma. J Androl 25: 922-925 